\title{
Semiovals from unions of conics
}

\author{
Jeremy M. Dover Keith E. Mellinger*
}

\begin{abstract}
A semioval in a projective plane $\pi$ is a collection of points $\mathcal{O}$ with the property that for every point $P$ of $\mathcal{O}$, there exists exactly one line of $\pi$ meeting $\mathcal{O}$ precisely in the point $P$. There are many known constructions of and theoretical results about semiovals, especially those that contain large collinear subsets.

In a Desarguesian plane $\pi$ a conic, the set of zeroes of some nondegenerate quadratic form, is an example of a semioval of size $q+1$ that also forms an arc (i.e., no three points are collinear). As conics are minimal semiovals, it is natural to use them as building blocks for larger semiovals. Our goal in this work is to classify completely the sets of conics whose union forms a semioval.
\end{abstract}

Keywords : semioval, conic

MSC 2000: 51E20

\section{Introduction}

Let $\operatorname{GF}(q)$ denote the finite field of order $q$, and let $\mathrm{GF}(q)^{*}$ denote the set of nonzero elements in this field. Let $\mathcal{P} \mathcal{G}(2, q)$ denote the finite Desarguesian projective plane over $\operatorname{GF}(q)$. A semioval in $\mathcal{P G}(2, q)$ is a collection of points $\mathcal{O}$ with the property that for every point $P$ of $\mathcal{O}$, there exists exactly one line meeting $\mathcal{O}$ precisely in the point $P$. A well-known construction for semiovals is the vertexless triangle, the set of points formed by the union of three non-concurrent lines with the intersection points removed. Many other constructions of semiovals can be formed by taking the vertexless triangle and then adding and removing

\footnotetext{
*Author acknowledges support by a Faculty Development Grant from the University of Mary Washington.
} 















\section{$1+0$ \\ are proportional.}
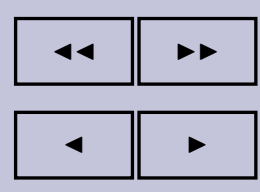

page $16 / 23$

go back

full screen

close

quit

We first wish to show that $c_{1}$, and thus $c_{2}$ must be zero. Proceeding by contradiction assume that $c_{1} \neq 0$, allowing us to normalize $c_{1}=1$ by absorbing the value into $\lambda_{1}$. If $c_{1}=1$, then we cannot have $a_{1}=b_{1}=0$ since that would give (by Polynomial (1)) $\mathcal{D}=V\left(x y+\left(1+\lambda_{1}\right) z^{2}\right.$ ), which forces $\mathcal{D}$ to be in the pencil generated by $\mathcal{C}_{1}$ and $\mathcal{C}_{2}$. Hence Polynomial (1) has at least one of the $x z$ and $y z$ cross-terms nonzero. This forces Polynomial (2) to have at least one of its $x z$ and $y z$ cross-terms nonzero, which implies $c_{2} \neq 0$ and at least one of $a_{2}$ and $b_{2}$ is nonzero. As above, since $c_{2} \neq 0$, we may assume without loss of generality that $c_{2}=1$.

Expanding Polynomials (1) and (2) and equating the coefficients with a constant of proportionality $\mu$, we obtain the following six equations:

$$
\begin{aligned}
\lambda_{1} a_{1}^{2} & =\mu\left(\lambda_{2} a_{2}^{2}\right), \\
1+2 \lambda_{1} a_{1} b_{1} & =\mu\left(1+2 \lambda_{2} a_{2} b_{2}\right), \\
\lambda_{1} b_{1}^{2} & =\mu\left(\lambda_{2} b_{2}^{2}\right), \\
2 \lambda_{1} a_{1} & =\mu\left(2 \lambda_{2} a_{2}\right), \\
2 \lambda_{1} b_{1} & =\mu\left(2 \lambda_{2} b_{2}\right), \\
1+\lambda_{1} & =\mu\left(k+\lambda_{2}\right) .
\end{aligned}
$$

Equations (6) and (7) let us solve for $a_{1}$ and $b_{1}$ in terms of $a_{2}$ and $b_{2}$ respectively, yielding $a_{1}=\frac{\mu \lambda_{2}}{\lambda_{1}} a_{2}$ and $b_{1}=\frac{\mu \lambda_{2}}{\lambda_{1}} b_{2}$. Plugging these into Equations (3) and (5) respectively, shows that $\frac{\mu \lambda_{2}}{\lambda_{1}} a_{2}^{2}=a_{2}^{2}$ and $\frac{\mu \lambda_{2}}{\lambda_{1}} b_{2}^{2}=b_{2}^{2}$. Since we cannot have both $a_{2}=0$ and $b_{2}=0$ we must have $\mu \lambda_{2}=\lambda_{1}$. Utilizing this fact in Equations (6) and (7) shows that $a_{1}=a_{2}$ and $b_{1}=b_{2}$, and in Equation (8) shows that $\mu k=1$. But plugging $\mu \lambda_{2}=\lambda_{1}, a_{1}=a_{2}$ and $b_{1}=b_{2}$ into Equation (4) forces $\mu=1$. Thus we must have $k=1$ implying $\mathcal{C}_{1}$ and $\mathcal{C}_{2}$ are identical, which is our contradiction.

Hence $c_{1}=0$, from which it follows that $c_{2}$ must also be zero. We claim $b_{1}$ and $b_{2}$ are both nonzero. First note that if $b_{1}$ is zero then Polynomial (1) has no $y^{2}$ term, which forces $b_{2}$ to be zero as well, and vice versa, so either both $b_{1}$ and $b_{2}$ are zero, or neither are. In the former case, the coefficients of $z^{2}$ in the Polynomials (1) and (2) are 1 and $k$ respectively, while the coefficients of $x y$ are both 1 ; since the polynomials are proportional this forces $k=1$, which yields the same contradiction as before. Thus $b_{1}$ and $b_{2}$ are both nonzero and we may normalize so that $b_{1}=b_{2}=1$. We again expand our polynomials and equate coefficients with a constant of proportionality $\mu$ to obtain four equations (since 
the $x z$ and $y z$ cross-terms have coefficient zero):

$$
\begin{aligned}
\lambda_{1} a_{1}^{2} & =\mu \lambda_{2} a_{2}^{2}, \\
1+2 \lambda_{1} a_{1} & =\mu\left(1+2 \lambda_{2} a_{2}\right), \\
\lambda_{1} & =\mu \lambda_{2}, \\
1 & =\mu k .
\end{aligned}
$$

From Equation (12) we have $\mu=\frac{1}{k}$, which plugged into Equation (11) yields $\lambda_{2}=k \lambda_{1}$. Combined with Equation (9) we obtain $a_{1}^{2}=a_{2}^{2}$. If $a_{1}=a_{2}$, then Equation (10) forces $\mu=1$ and thus $k=1$, which is again false. If $a_{1}=-a_{2}$, Equation (10) yields $k\left(1+2 \lambda_{1} a_{1}\right)=1-2 k \lambda_{1} a_{1}$, which we simplify to obtain $\lambda_{1} a_{1}=\frac{1-k}{4 k}$.

At this point, we have shown that if there exists a conic mutually interior to $\mathcal{C}_{1}$ and $\mathcal{C}_{2}$ but not in the pencil they generate, it must be of the form $\mathcal{D}=$ $V\left(x y+z^{2}+\lambda(a x+y)^{2}\right)$, or alternatively $\mathcal{D}=V\left(x y+k z^{2}+k \lambda(-a x+y)^{2}\right)$, where $\lambda a=\frac{1-k}{4 k}$. We now need to determine which conics $\mathcal{D}$ of this form are in fact mutually interior with both $\mathcal{C}_{1}$ and $\mathcal{C}_{2}$, which involves showing that $\mathcal{D}$ contains no exterior point of $\mathcal{C}_{1}$ or $\mathcal{C}_{2}$, and vice versa. Suppose $\left(y_{0}, y_{1}, y_{2}\right)$ is a point of $\mathcal{D}=V\left(x y+z^{2}+\lambda(a x+y)^{2}\right)$. Appealing to Lemma 3.2 as before $\left(y_{0}, y_{1}, y_{2}\right)$ is exterior to $\mathcal{C}_{1}$ if $-\frac{1}{4} \lambda\left(a y_{0}+y_{1}\right)^{2}$ is a nonzero square, which occurs if and only if $-\lambda$ is a nonzero square. Hence $\mathcal{D}$ contains no exterior point of $\mathcal{C}_{1}$ if and only if $-\lambda$ is a nonsquare. Repeating the calculation for the other three cases shows that

1. $\mathcal{C}_{1}$ contains no exterior point of $\mathcal{D}$ if and only if $\frac{\lambda}{k}$ is a nonsquare.

2. $\mathcal{D}$ contains no exterior point of $\mathcal{C}_{2}$ if and only if $k \lambda$ is a nonsquare.

3. $\mathcal{C}_{2}$ contains no exterior point of $\mathcal{D}$ if and only if $k \lambda$ is a nonsquare.

When $q \equiv 1$ (mod 4$)$ both -1 and $k$ are nonzero squares, so these conditions are satisfied if and only if $\lambda$, and thus $-\lambda$, is a nonsquare. When $q \equiv 3(\bmod 4)$ both -1 and $k$ are nonsquares, implying these conditions are met if and only if $\lambda$ is a nonzero square, or equivalently $-\lambda$ is a nonsquare. This proves the result.

Theorem 5.4 limits the number and structure of conics mutually interior to both $\mathcal{C}_{1}$ and $\mathcal{C}_{2}$, allowing us to press on to determine if there are any sets of four non-copencilar conics that are mutually interior. There are two possible configurations of such conics: $\mathcal{C}_{1}, \mathcal{C}_{2}$ and $\mathcal{C}_{3}$ mutually interior in a pencil, with $\mathcal{C}_{4}$ not in the pencil they generate, or no three of the conics in a common pencil. We first show that the former case can never happen. 



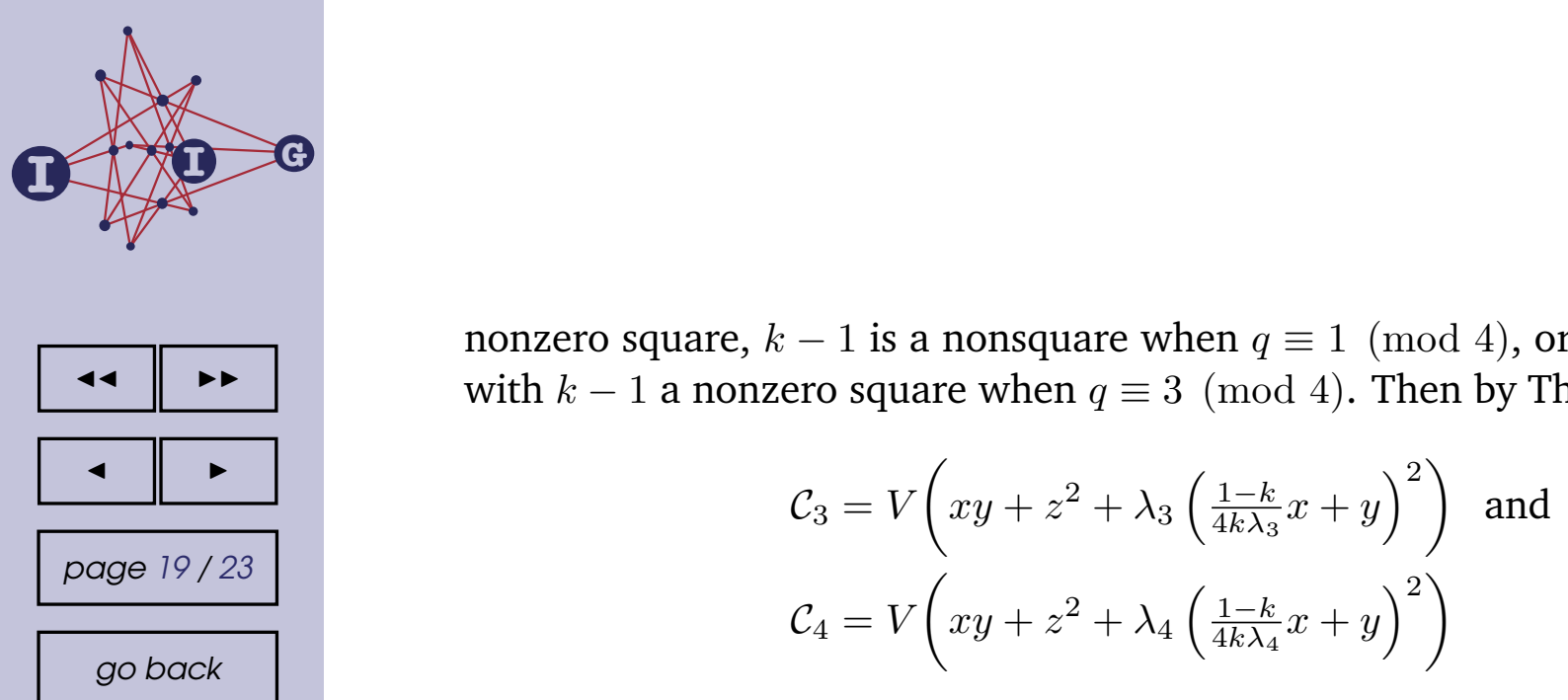

for some $\lambda_{3}, \lambda_{4} \in \mathrm{GF}(q)^{*}$ such that $-\lambda_{3},-\lambda_{4}$ are nonsquares.

The conics $\mathcal{C}_{3}$ and $\mathcal{C}_{4}$ are also mutually interior, so Corollary 3.4 implies that $\mathcal{C}_{4}$ can also be written as

$$
V\left(x y+z^{2}+\lambda_{3}\left(\frac{1-k}{4 k \lambda_{3}} x+y\right)^{2}+\chi(a x+b y+c z)^{2}\right)
$$

for some $\chi \in \mathrm{GF}(q)^{*}$ and $a, b, c \in \mathrm{GF}(q)$. Hence the polynomials

$$
x y+z^{2}+\lambda_{4}\left(\frac{1-k}{4 k \lambda_{4}} x+y\right)^{2}
$$

and

$$
x y+z^{2}+\lambda_{3}\left(\frac{1-k}{4 k \lambda_{3}} x+y\right)^{2}+\chi(a x+b y+c z)^{2}
$$

are proportional.

Note that Polynomial (13) has no $x z$ and $y z$ cross terms, so the proportionality shows that Polynomial (14) also has zero coefficients for its $x z$ and $y z$ cross-terms. Therefore either $c=0$, or $a=b=0$; we first show that $c$ cannot be zero. Assume by way of contradiction that $c=0$. Expanding Polynomials (13) and (14) and equating the coefficients with a constant of proportionality $\mu$, we obtain the following four equations:

$$
\begin{aligned}
\frac{(1-k)^{2}}{16 k^{2} \lambda_{4}} & =\mu\left(\frac{(1-k)^{2}}{16 k^{2} \lambda_{3}}+\chi a^{2}\right), \\
\frac{1-k}{2 k}+1 & =\mu\left(1+\frac{1-k}{2 k}+2 \chi a b\right), \\
\lambda_{4} & =\mu\left(\lambda_{3}+\chi b^{2}\right) \\
1 & =\mu .
\end{aligned}
$$

Equation (18), equating the $z^{2}$ coefficients, shows immediately that $\mu=1$. Equation (17) then shows that $\lambda_{4}=\lambda_{3}+\chi b^{2}$; since $\lambda_{3}$ and $\lambda_{4}$ being equal would force $\mathcal{C}_{3}=\mathcal{C}_{4}$, we must have $\chi b^{2} \neq 0$ implying $b \neq 0$. On the other hand Equation (16) shows $2 \chi a b=0$, which forces $a=0$. Finally we plug $a=0$ and 


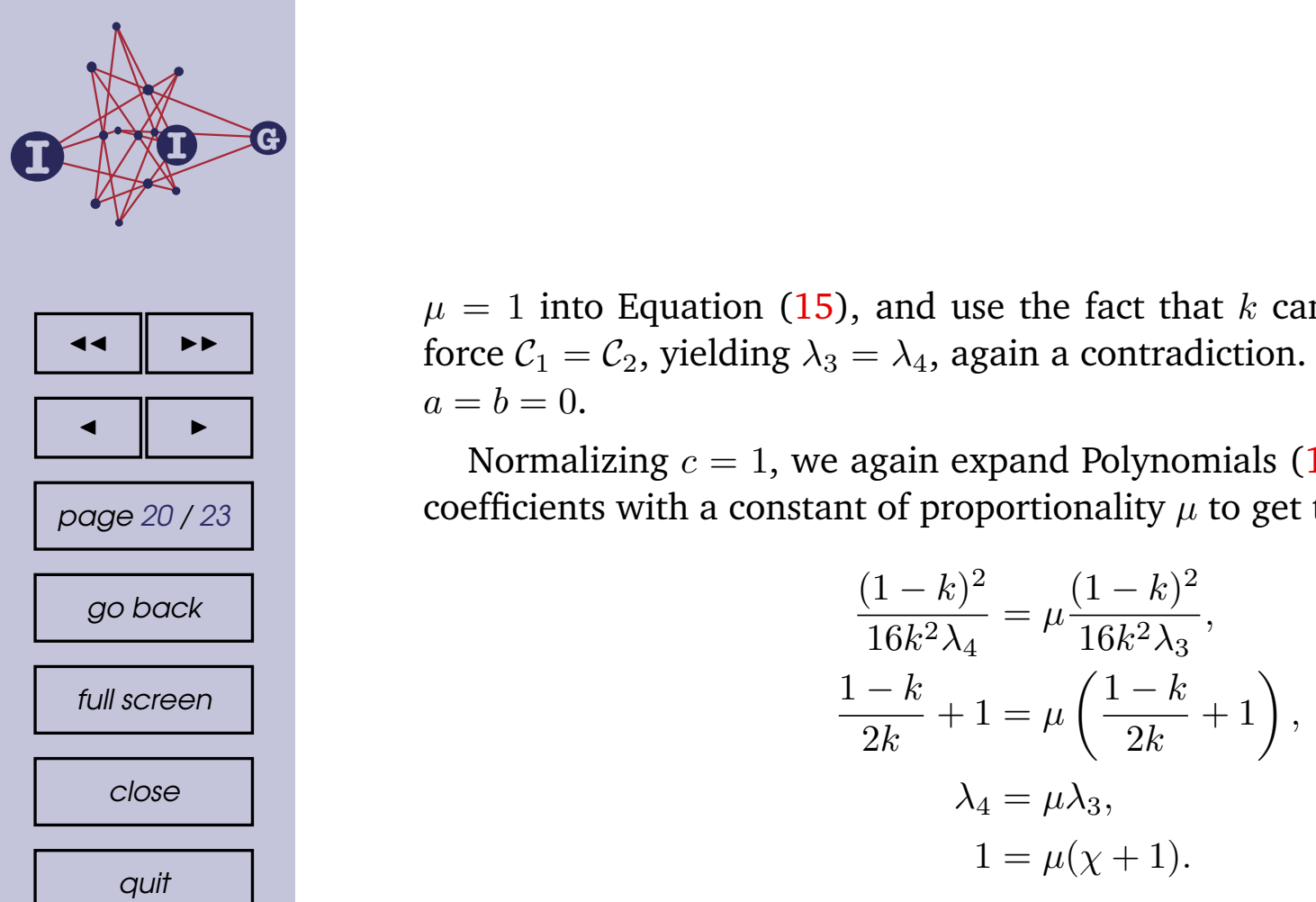

$\mu=1$ into Equation (15), and use the fact that $k$ cannot be 1 as that would force $\mathcal{C}_{1}=\mathcal{C}_{2}$, yielding $\lambda_{3}=\lambda_{4}$, again a contradiction. Hence $c$ is nonzero, and $a=b=0$.

Normalizing $c=1$, we again expand Polynomials (13) and (14) and equate coefficients with a constant of proportionality $\mu$ to get the following equations:

$$
\begin{aligned}
\frac{(1-k)^{2}}{16 k^{2} \lambda_{4}} & =\mu \frac{(1-k)^{2}}{16 k^{2} \lambda_{3}}, \\
\frac{1-k}{2 k}+1 & =\mu\left(\frac{1-k}{2 k}+1\right), \\
\lambda_{4} & =\mu \lambda_{3}, \\
1 & =\mu(\chi+1) .
\end{aligned}
$$

Examining Equation (20), we see immediately that either $\mu=1$ or $\frac{1-k}{2 k}+1=0$. However $\mu=1$ combined with Equation (21) would force $\lambda_{3}=\lambda_{4}$, yet another contradiction, so we must have $\frac{1-k}{2 k}+1=0$ which forces $k=-1$. Equation (19) then yields $\lambda_{3}=\mu \lambda_{4}$, which combined with Equation (21) shows that $\mu=-1$. Finally from Equation (22) we determine that $\chi=-2$.

First notice that $\lambda_{3}$ and $\lambda_{4}$ are opposites, which means that for $q \equiv 3(\bmod 4)$ $\lambda_{3}$ and $\lambda_{4}$ have opposite quadratic character, meaning not both $-\lambda_{3}$ and $-\lambda_{4}$ are nonsquares. Hence no such mutually interior conics $\mathcal{C}_{3}$ and $\mathcal{C}_{4}$ can exist when $q \equiv 3(\bmod 4)$. Assuming $q \equiv 1(\bmod 4)$, we must check the constraints on $k=-1$, namely that $k$ is a nonzero square and $k-1$ is a nonsquare. The former condition is clearly true, but the latter is only true when $k-1=-2$ is a nonsquare, or equivalently when 2 is a nonsquare, which occurs only for $q \equiv 5$ $(\bmod 8)$.

Thus only in the case where $q \equiv 5(\bmod 8)$ is it possible that a set of four non-copencilar, mutually interior conics can exist, and if it exists the conics in the set must be isomorphic to $\mathcal{C}_{1}=V\left(x y+z^{2}\right), \mathcal{C}_{2}=V\left(x y-z^{2}\right), \mathcal{C}_{3}=$ $V\left(x y+z^{2}+\lambda\left(\frac{-1}{2 \lambda} x+y\right)^{2}\right)$ and $\mathcal{C}_{4}=V\left(x y+z^{2}-\lambda\left(\frac{1}{2 \lambda} x+y\right)^{2}\right)$ for some nonsquare $\lambda$. However we need to check that these conics are actually mutually interior. We can use Theorems 4.1 and 5.4 to show all pairs are mutually interior, except $\mathcal{C}_{3}$ and $\mathcal{C}_{4}$. Using Lemma 3.2 as in Theorem 5.4 quickly shows that $\mathcal{C}_{3}$ and $\mathcal{C}_{4}$ are mutually interior if $\frac{1}{2}$ is a nonsquare, which is true when $q \equiv 5(\bmod 8)$. Thus $\mathcal{C}_{1}, \mathcal{C}_{2}, \mathcal{C}_{3}$ and $\mathcal{C}_{4}$ are a set of non-copencilar mutually interior conics when $q \equiv 5(\bmod 8)$, and uniqueness of $\mathcal{C}_{4}$ given $\mathcal{C}_{1}, \mathcal{C}_{2}$ and $\mathcal{C}_{3}$ follows immediately from the form of $\mathcal{C}_{4}$. 


\section{Conclusion}

We summarize the sets of mutually interior conics in $\mathcal{P G}(2, q)$ in Table 1 .

While the semiovals discussed in this paper are interesting in their own right, we are very interested in finding examples of blocking semiovals, i.e., semiovals that are also blocking sets. While some of the sets we constructed here are blocking semiovals in small order planes, the only infinite family arises from the tangent pencils discovered by Szőnyi [8]. However we are hopeful of using our results here to find blocking semiovals that contain some of the sets constructed in this paper.

The authors would like to take this opportunity to thank the reviewers and quit editors for their many useful comments and constructive criticism. 
Table 1: Sets of mutually interior conics

\begin{tabular}{|l|c|c|c|c|}
\hline canonical forms & restrictions & \# of conics & pencil structure & intersections \\
\hline$x y+z^{2}$ & all $q$ & 1 & n/a & n/a \\
\hline$\left\{x y+a_{i} z^{2}\right\}$ & $q \equiv 1(\bmod 4)$ & $\leq \sqrt{q}$ & $\begin{array}{c}\text { copencilar } \\
\text { copencilar }\end{array}$ & secant \\
& $q \equiv 3(\bmod 4)$ & 2 & secant \\
\hline$\left\{x^{2}-y z-a_{i} z^{2}\right\}$ & $q \equiv 1(\bmod 4)$ & $\leq \sqrt{q}$ & copencilar & tangent \\
\hline$\left\{x^{2}-s y^{2}+a_{i} z^{2}\right\}$ & $q \equiv 1(\bmod 4)$ & $\leq \sqrt{q}-1$ & copencilar & disjoint \\
$s$ nonsquare & $q \equiv 3(\bmod 4)$ & 2 & copencilar & disjoint \\
\hline $\begin{array}{l}x y+z^{2}, x y+k z^{2}, \\
x y+z^{2}+\lambda\left(\frac{1-k}{4 k \lambda} x+y\right)^{2}\end{array}$ & $q$ odd & 3 & non-copencilar & pairwise secant \\
\hline $\begin{array}{l}x y+z^{2}, x y-z^{2}, \\
x y+z^{2}+\lambda\left(\frac{-1}{2 \lambda} x+y\right)^{2},\end{array}$ & $q \equiv 5(\bmod 8)$ & 4 & non-copencilar & pairwise secant \\
$x y+z^{2}-\lambda\left(\frac{1}{2 \lambda} x+y\right)^{2}$ & & & & \\
\hline
\end{tabular}




\section{References}

[1] V. Abatangelo, J.C. Fisher, G. Korchmaros and B. Larato, On the mutual position of two irreducible conics in PG(2,q), $q$ odd, Adv. Geom. 11 (2011), 603-614.

[2] H.S.M. Coxeter, "Projective geometry", Blaisdell Publishing Co. Ginn and Co. New York-London-Toronto, 1964.

[3] L.E. Dickson, On families of quadratic forms in a general field, Quarterly J. Pure Appl. Math. 39 (1908), 316-333.

[4] J. W. P. Hirschfeld, "Projective geometries over finite fields", Oxford Mathematical Monographs, The Clarendon Press Oxford University Press, New York, second edition, 1998.

[5] G. Kiss, A survey on semiovals, Contrib. Discrete Math. 3 (2008), 81-95.

[6] G. Kiss, S. Marcugini and F. Pambianco, On the spectrum of the sizes of semiovals in PG(2, q), q odd, Discrete Math. 310 (2010), 3188-3193.

[7] B. Segre, Proprietà elementari relative ai segmenti ed alle coniche sopra un campo qualsiasi ed una congettura di Seppo Ilkka per il caso dei campi di Galois, Ann. Mat. Pura Appl. (4) 96 (1972), 289-337.

[8] T. Szőnyi, Note on the existence of large minimal blocking sets in Galois planes, Combinatorica 12 (1992), 227-235.

Jeremy M. Dover

Dover Networks LLC, 445 Poplar LEAF Dr., EdGEWATER, MD 21037

e-mail: jeremy@dovernetworks.com

Keith E. Mellinger

Department of Mathematics, University of Mary Washington, 1301 College Avenue, FredERICKSBURG, VA 22401-5300

e-mail: kmelling@umw.edu 